\title{
Superdense Stellar Configurations in the Bimetric Scalar-Tensor Theory of Gravity
}

\author{
L. Sh. Grigorian ${ }^{1}$, H. F. Khachatryan ${ }^{1}$, A. A. Saharian ${ }^{1,2 *}$ \\ ${ }^{1}$ Institute of Applied Problems in Physics NAS RA, 25 Nersessian Street, 0014 Yerevan, \\ Armenia \\ ${ }^{2}$ Department of Physics, Yerevan State University, 1 Alex Manoogian Street, 0025 Yerevan, \\ Armenia \\ *E-mail:saharian@ysu.am
}

\begin{abstract}
Models of static spherically-symmetric stellar configurations are discussed within the framework of the Bimetric scalar-tensor theory of gravity. The latter, in addition to the metric tensor and the scalar field, contains a background metric tensor as an absolute variable of the theory. The simplest variant of the theory with a constant coupling parameter and with a zero cosmological function is considered. The analysis includes both the white dwarfs and neutron stars. It is shown that, depending on the value of the theory parameter, the corresponding masses can be notably larger than those in general relativity.
\end{abstract}

Keywords: scalar-tensor theories - bimetric theories - superdense stars.

\section{Introduction}

The scalar-tensor theories are among the most popular alternatives of general relativity (for reviews see Will (2018), Fujii \& Maeda (2009)). The gravitational sector in this theories, in addition to the metric tensor contains a scalar field, usually referred as a gravitational scalar. The recent interest to scalar-tensor theories is related to the fact that a scalar field directly interacting with curvature of the spacetime appear in a number of fundamental theories. The latter include Kaluza-Klein type theories with extra spatial dimensions, supergravity and superstring theories. The scalar fields also play an important role in recent cosmology. In particular, in a large number of inflationary models the accelerated expansion of the Universe at early stages of the evolution is driven by the stress-energy of a scalar field (inflaton). In a class of cosmological models, scalar fields are responsible for accelerated expansion of the Universe at recent epoch. The scalar-tensor theories may 
also provide alternative models for dark matter and dark energy. Another class of modified gravity theories, the so-called $f(R)$-gravities (for recent reviews in various types of modified gravity theories see Will (2018), Clifton et al. (2012), Schmidt-May \& von Strauss (2016)), are presented in the form of a scalar-tensor theory with the potential for a scalar field determined by the $f(R)$ function in the gravitational Lagrangian density.

The bimetric (or tensor-tensor) theories present another class of theories alternative to general relativity. In these theories the additional field is a second rank tensor. The latter can be either dynamical or non-dynamical (absolute). Among the first examples for bimetric theories is the Rosen theory. Recent activity in considering theories of gravity with two metric tensors is partially related to non-linear extensions of Fierz-Pauli massive gravity (for a review see Hinterbichler (2012)). In these models the second tensor is required for the construction of non-linear generalizations of the Fierz-Pauli mass term.

In Grigorian \& Saharian (1990a, 1990b, 1990c, 1991) we have suggested a variant of scalar-tensor theories involving a second non-dynamical metric tensor: bimetric scalar-tensor theory (BSTT). The BSTT belongs to the class of

metric theories of gravity and consequently obeys the Einstein equivalence principle (for a general review see Will (2018)). Here we consider a static, spherically symmetric configuration of gravitating masses within the framework of BSTT. In a variant of the theory with a constant coupling function and zero cosmological function the corresponding solution outside the matter distribution (external solution) has been found in Grigorian \& Saharian (1990b). The results for a numerical integration of the internal equations were presented in Grigorian \& Saharian (1990b) and Grigorian et al. (2018) and Avakian et al. (1991) for some special cases of the equation of state for gravitating masses.

\section{BSTT action and the field equations}

The BSTT belongs to the class of metric theories of gravity with a preferred geometry. In addition to the curved metric tensor $g_{i k}$ it contains a dynamical scalar field $\varphi(x)$ and a nondynamical metric $\gamma_{i k}$. The latter is the absolute variable of the theory. We will denote by $\Gamma_{i k}^{l}$ and $\hat{\Gamma}_{i k}^{l}$ the Cristoffel symbols for the metrics $g_{i k}$ and $\gamma_{i k}$, respectively. Though these quantities are not tensors, their difference, $\bar{\Gamma}_{i k}^{l}=\Gamma_{i k}^{l}-\hat{\Gamma}_{i k}^{l}$, is a tensor (affine deformation tensor). It can be written as

$$
\bar{\Gamma}_{i k}^{l}=\frac{1}{2} g^{l m}\left(\hat{\nabla}_{i} g_{m k}+\hat{\nabla}_{k} g_{m i}-\hat{\nabla}_{m} g_{i k}\right)
$$

where $\hat{\nabla}_{i}$ stands for the covariant derivative with respect to the metric $\gamma_{i k}$. As a consequence of the latter property, in theories with two metrics it is 
possible to construct a Lagrangian density for the gravitational field from the metric tensor and its first derivatives. In the bimetric formulation of general relativity, up to a coefficient, the corresponding scalar is given by

$$
\Lambda_{g}=g^{i k}\left(\bar{\Gamma}_{i n}^{l} \bar{\Gamma}_{k l}^{n}-\bar{\Gamma}_{i k}^{l} \bar{\Gamma}_{n l}^{n}\right) .
$$

This scalar differs from the Ricci scalar $R$ for the metric $g_{i k}$ by a total divergence, $R=\Lambda_{g}+\nabla_{l} \bar{w}^{l}$ with $\bar{w}^{l}=g^{i k} \bar{\Gamma}_{i k}^{l}-g^{l k} \bar{\Gamma}_{i k}^{i}$. As a consequence of the latter property, the field equations obtained from (2) coincide with the Einstein equations for the gravitational field in general relativity. By the standard variational procedure with respect to the background metric $\gamma_{i k}$, from (2) we can construct the energy-momentum tensor for the gravitational field (see, for example, Babak \& Grishchuk (1999)).

The BSTT is constructed on the base of general relativity in bimetric formulation in a way similar to that the usual scalar-tensor theories are constructed from general relativity with the Lagrangian density proportional to the Ricci scalar. The action of the theory, in its general form, reads (here and below we use the units $c=1$ )

$$
S=\int d^{4} x \sqrt{-g}\left[-\frac{\varphi}{2} \Lambda_{g}+\frac{\zeta(\varphi)}{2 \varphi} g^{i k} \partial_{i} \varphi \partial_{k} \varphi-\Lambda(\varphi)+L_{m}\left(g_{i k}, q_{a}, \nabla_{l} q_{a}\right)\right]
$$

where $\zeta(\varphi)$ is a dimensionless coupling function, $\Lambda(\varphi)$ is the cosmological function, $L_{m}$ is the Lagrangian density for nongravitational fields collectively denoted by $q_{a}, \nabla_{l}$ is the covariant derivative operator with respect to the metric $g_{i k}$. The simplest variant of the theory corresponds to a constant coupling function $\zeta(\varphi)=\zeta=$ const and to the zero cosmological function $\Lambda(\varphi)=0$. In the nongravitational part of the Lagrangian density the gravitational field enters through the metric tensor $g_{i k}$ only and, hence, the theory obeys the Einstein equivalence principle. In usual scalar-tensor theories instead of $\Lambda_{g}$ in (3) the scalar curvature $R$ for the metric tensor $g_{i k}$ appears. Though the difference $R-\Lambda_{g}$ is a total derivative, because of the spacetime dependence of $\varphi$, the field equations following from (3) differ from those for scalar-tensor theories.

The field equations for the metric tensor and the scalar field, obtained from (3), have the form

$$
\begin{aligned}
\varphi R_{i k}+\bar{\Gamma}_{i k}^{l} \partial_{l} \varphi-\bar{\Gamma}_{l(k}^{l} \partial_{i)} \varphi-\zeta(\varphi) \partial_{i} \varphi \partial_{k} \varphi / \varphi & =T_{i k}-g_{i k} T / 2-\Lambda(\varphi) g_{i k}, \\
2 \zeta(\varphi) \nabla_{l} \nabla^{l} \varphi+\left[\zeta^{\prime}(\varphi)-\zeta(\varphi) / \varphi\right] \nabla_{l} \varphi \nabla^{l} \varphi & =-\varphi\left[2 \Lambda^{\prime}(\varphi)+\Lambda_{g}\right],
\end{aligned}
$$

where $T_{i k}=(2 / \sqrt{-g}) \delta\left(\sqrt{-g} L_{m}\right) / \delta g^{i k}$ is the metric energy-momentum tensor for the nongravitational matter, $T=g^{m n} T_{m n}$, the prime means the derivative with respect to the field $\varphi$ and the brackets in the index expression of the first equation mean the symmetrization with respect to the 
indices included. By using the first equation in (4), the equation for the scalar field can also be written in the form

$$
\nabla_{l}\left[2 \zeta(\varphi) \nabla^{l} \varphi-\varphi \bar{w}^{l}\right]-\zeta^{\prime}(\varphi) \nabla_{l} \varphi \nabla^{l} \varphi=T+4 \Lambda(\varphi)-2 \varphi \Lambda^{\prime}(\varphi) .
$$

From the equation for the nongravitational matter, $\delta L_{m} / \delta q_{a}=0$, it follows that $\nabla_{k} T_{i}^{k}=0$. Note that, unlike to the usual scalar-tensor theories, in BSTT the latter equation does not follow from the field equations. This is a consequence of the presence of nondynamical metric $\gamma_{i k}$. Another consequence is that in BSTT the gravitational field is characterized by the energy-momentum tensor (Grigorian \& Sahakian 1990d, 1994).

The first step to check the validity of the gravitational theory is to consider the Newtonian and post-Newtonian approximations and to compare the predictions of the theory with the observational data, in particular, for the gravitational effects in the solar system and in double pulsar systems. The gravitational effects are expressed in terms of the PPN (parametrized post-Newtonian) parameters of the theory. Under the condition $\left|\zeta\left(\varphi_{0}\right)\right| \gg v^{2}$ the PPN parameters of BSTT coincide with those for general relativity. Here $\varphi=\varphi_{0}=(8 \pi G)^{-1}$ is the value for the gravitational scalar at the recent stage of the Universe expansion, $G$ is the Newtonian gravitational constant, $v^{2} \sim P / \rho\left(P / \rho \sim 10^{-5}\right.$ in the solar system $)$ is the velocity in a post-Newtonian system with the pressure $P$ and the energy density $\rho$. In particular, in contrast to most other bimetric theories, the PPN parameters of BSTT do not depend on the cosmological connection coefficients. This is related to the fact that the background metric $\gamma_{i k}$ enters in the action of the theory through its Cristoffel symbols only. Note that in the Brans-Dicke theory (the simplest variant of scalar-tensor theories with $\zeta(\varphi)=\zeta=$ const and $\Lambda(\varphi)=0)$ for the post-newtonian parameter $\gamma$ one has $\gamma=(1+\zeta) /(2+\zeta)$ and the observational data within the framework of the solar system strongly constrain (mainly from Cassini measurements of the Shapiro time delay) the theory parameter: $\zeta>4 \cdot 10^{4}$. This difference between the two scalar-tensor theories is related to that in the Brans-Dicke theory the scalar field is sourced by the scalar curvature $R$ (in the equation for the scalar field, that is the analog of the second equation in (4), $R$ stands instead of $\Lambda_{g}$ ) which is of the order $v^{2}$ and, hence, the same for the variations of scalar field. In BSTT, the scalar field is sourced by $\Lambda_{g}$ which has the order $v^{4}$ and related to that the variations of scalar field in post-Newtonian systems are of the order $v^{4}$ if the theory parameter is not too small (in the variations the parameter $\zeta$ enters in the form $1 / \zeta$ (see, for example, (19) below)).

The gravitational waves in BSTT have been analyzed in Saharian (1993a, 1993b). In this theory, the velocity of weak perturbations of the curved metric coincides with the speed of light in the vacuum. In a variant of the theory with zero cosmological function, the same is the case for the scalar field. Similar to general relativity, the BSTT is of class $N_{2}$ in $E(2)$ classification of 
the gravitational waves polarization of metric theories of gravity. In particular, this means that in BSTT the weak perturbations for metric and scalar field propagate independently. In usual scalar-tensor theories (for example, in Brans-Dicke theory) the equation for weak perturbations of the metric tensor contain a contribution from the scalar field as well (see, for example, Will (2018)). As a consequence of that the scalar and metric perturbations are mixed and the theory belongs to the class $N_{3}$. Related to the presence of a scalar degree of freedom, in addition to the standard quadrupole gravitational radiation, in BSTT there is also dipole gravitational radiation. The corresponding Peters-Mathews parameters for the radiation from a gravitating system with small velocities and nonrelativistic internal structure have been determined in Saharian (1993a). The corresponding formalism for a system of gravitating bodies with small velocities but with relativistic internal structure (modified Einstein-Infeld-Hoffmann formalism) has been considered in Saharian (1993b).

\section{Spherically symmetric static configurations}

Consider a static spherically symmetric stellar configuration described by the energy-momentum tensor

$$
T_{i k}=(\rho+P) u_{i} u_{k}-P g_{i k} .
$$

Here $\rho$ and $P$ are the energy density and the pressure of the matter and $u_{i}$ is the corresponding four-velocity. In the coordinate system $(t, r, \theta, \phi)$ with the background metric

$$
\gamma_{i k}=\operatorname{diag}\left(1,-1,-r^{2},-r^{2} \sin ^{2} \theta\right),
$$

the curved metric tensor is presented as

$$
g_{i k}=\operatorname{diag}\left(e^{\nu},-e^{\lambda},-r^{2} e^{\mu},-r^{2} e^{\mu} \sin ^{2} \theta\right),
$$

where $\nu(r), \lambda(r), \mu(r)$ are functions of the radial coordinate tending to zero at large distances from the configuration. The scalar field depends on the radial coordinate alone: $\varphi=\varphi(r)$. We will consider the variant of the theory with $\zeta(\varphi)=\zeta=$ const and $\Lambda(\varphi)=0$.

From the equation (4), for $0 \leqslant r<\infty$ we get

$$
\left(1-e^{\lambda-\mu}\right)\left[\varphi^{\prime \prime}+\varphi^{\prime}\left(\frac{2}{r}+\frac{\nu^{\prime}+\lambda^{\prime}}{2}\right)\right]=\varphi^{\prime}\left(\lambda^{\prime}-\mu^{\prime}\right),
$$

where the prime stands for the derivative with respect to $r$. The integration of this equation leads to the result

$$
\left(e^{\mu-\lambda}-1\right) r^{2} \varphi^{\prime} e^{(\nu+\lambda) / 2}=\text { const. }
$$


Assuming that $\varphi^{\prime}(0)$ is finite from here we find $\mu(r)=\lambda(r)$. With this relation, the spatial part of the metric tensor (8) is conformally flat (this corresponds to isotropic coordinates in general relativity). Introducing the notation $z=(\nu+\lambda) / 2$ and the functions

$$
M(r)=4 \pi \int_{0}^{r} d r r^{2}(\rho+3 P) e^{3 z-\nu}, \beta(r)=4 \pi \int_{0}^{r} d r r^{2} P e^{3 z-\nu},
$$

the remaining equations are presented in the form

$$
\begin{aligned}
\nu^{\prime} & =\frac{M(r) e^{-z}}{4 \pi r^{2} \varphi}, \zeta \frac{\varphi^{\prime}}{\varphi}+2 z^{\prime}=\frac{3 \beta(r) e^{-z}}{4 \pi r^{2} \varphi} \\
P^{\prime} & =-\frac{\nu^{\prime}}{2}(\rho+P), z^{\prime 2}+\frac{2}{r} z^{\prime}-\frac{\nu^{\prime 2}}{4}-\frac{\zeta}{2}\left(\frac{\varphi^{\prime}}{\varphi}\right)^{2}=\frac{P}{\varphi} e^{2 z-\nu},
\end{aligned}
$$

In addition, the equation of state $\rho=\rho(P)$ should be given. For nongravitational sources obeying the strong energy condition one has $\rho+3 P \geqslant 0$ and the function $\nu(r)$ is monotonically increasing.

Let us denote by $r_{1}$ the radius of the configuration. Then, in the region $r>r_{1}$ one has $\rho=0=P$. In this region $M(r)=M\left(r_{1}\right) \equiv M, \beta(r)=$ $\beta\left(r_{1}\right) \equiv \beta$ and $\varphi(r) \rightarrow \varphi_{0}=1 /(8 \pi G)$ for $r \rightarrow \infty$. The latter condition is required to ensure the limiting transition to the Newtonian gravity for weak fields. Note that the Tolmen formula for the mass $M=M\left(r_{1}\right)$ of the configuration, obtained in general relativity, is valid in BSTT as well (see Avakian et al. (1991)). In the case $\zeta \neq 2$ the corresponding solution for the function $\nu=\nu(r)$ is given by

$$
e^{\nu}=\exp \left[\frac{1}{\sqrt{a}}\left(2 \arctan \frac{2 l-\alpha / \zeta}{\sqrt{a}}-\pi\right)\right],
$$

for $a \geqslant 0$ and by

$$
e^{\nu}=\left|\frac{2 l-\alpha / \zeta-\sqrt{-a}}{2 l-\alpha / \zeta+\sqrt{-a}}\right|^{\frac{1}{\sqrt{-a}}},
$$

for $a \leqslant 0$. Here $l=r / r_{g}, r_{g}=2 G M$, and

$$
\alpha=\frac{12 \pi}{M} \int_{0}^{r_{1}} d r r^{2} P e^{3 z-\nu}, a=\frac{1-\alpha^{2}}{2 \zeta}-\frac{1}{4} .
$$

For the remaining metric component and the scalar field we get the expressions

$$
\begin{aligned}
e^{\lambda} & =\left|\frac{4 l^{2}}{(2 l-\alpha / \zeta)^{2}+a}\right|^{\frac{2 \zeta}{2-\zeta}} \exp \left[\frac{\zeta+2(\alpha-1)}{2-\zeta} \nu\right], \\
\frac{\varphi}{\varphi_{0}} & =\left|\frac{4 l^{2}}{(2 l-\alpha / \zeta)^{2}+a}\right|^{\frac{2}{\zeta-2}} \exp \left(\frac{\alpha \nu}{\zeta-2}\right) .
\end{aligned}
$$


As seen, the external solution, in addition to the mass $M$, is determined by the parameter $\alpha$. In the special case $\zeta=2$ the solution is specified to

$$
\begin{aligned}
e^{\nu} & =|1-\alpha / 2 l|^{2 / \alpha}, e^{\lambda}=|1-\alpha / 2 l|^{(1-1 / \alpha)^{2}} \exp \left(\frac{1+1 / \alpha}{2 l}\right), \\
\frac{\varphi}{\varphi_{0}} & =|1-\alpha / 2 l|^{\frac{1}{2}(1-1 / \alpha)^{2}} \exp \left(-\frac{1+1 / \alpha}{4 l}\right) .
\end{aligned}
$$

Under the condition $-\zeta / 2 \leqslant \alpha^{2}<1-\zeta / 2$ the external solution is regular for all $r_{1}$. For the values of the parameter $\alpha$ outside this region the external solution is singular at

$$
l=l_{c}=\frac{1}{2}\left(\frac{\alpha}{\zeta}+\sqrt{\frac{1}{4}-\frac{1-\alpha^{2}}{2 \zeta}}\right),
$$

if $r_{1}<l_{c} r_{g}$. In the limit $\zeta \rightarrow \infty$ one has $a=-1 / 4$ and the solutions (14), (16) reduce to the Schwarzschild solution in general relativity in isotropic coordinates:

$$
e^{\nu} \rightarrow\left(\frac{l-1 / 4}{l+1 / 4}\right)^{2}, e^{\lambda} \rightarrow\left(1+\frac{1}{4 l}\right)^{4}, \varphi \rightarrow \varphi_{0}
$$

In these coordinates the horizon corresponds to the spherical surface $r=$ $r_{g} / 4$.

At large distances from the spherically symmetric configuration one has

$$
e^{\nu} \approx 1-\frac{r_{g}}{r}+\frac{1-\alpha / \zeta}{2}\left(\frac{r_{g}}{r}\right)^{2}, e^{\nu} \approx 1+\frac{r_{g}}{r}, \frac{\varphi}{\varphi_{0}} \approx 1-\frac{\alpha r_{g}}{\zeta r} .
$$

Similar to the post-Newtonian considerations, in the expansion for the $g_{00}$ component of the metric tensor we have also kept the next-to-leading correction. As seen, the theory parameter enters into the corrections in the form of the ratio $\alpha / \zeta$. For $\alpha \ll \zeta$ (this is the case for nonrelativistic sources and for $\zeta \gtrsim 1)$ the variation in the scalar field is much smaller than that for the metric tensor components. Note that in the solar system $\alpha \lesssim 5 \cdot 10^{-6}$. For this type of matter sources one has $l_{c} \approx(1 / 4) \sqrt{1-2 / \zeta}$ and the spherical surface $l=l_{c}$ is inside the Schwarzschild horizon in general relativity.

\section{Superdense stellar configurations}

In this section we consider models of superdense configurations based on the numerical integration of the equations (12). It is convenient to use the pressure as an independent variable and to consider the equation of state of the form $\rho=\rho(P)$. For a given value of the central pressure $P(0)$, the quantities $\nu(0), \lambda(0), \varphi(0)$ and the mass $M$ are determined by the matching 
conditions for the external and internal solutions on the surface of the gravitating body $r=r_{1}$, where $P\left(r_{1}\right)=0$. Figure 1 presents the dependence of the mass (in units of the solar mass $M_{\odot}$ ) of a superdense configuration as a function of $P(0)$ for different values of the theory parameter $\zeta$ (numbers near the curves). In the numerical integration we have used the equation of state from Sahakian (1974), Grigorian (1983), and Sahakian (1995). The curves with $\zeta=\infty$ correspond to configurations in general relativity.

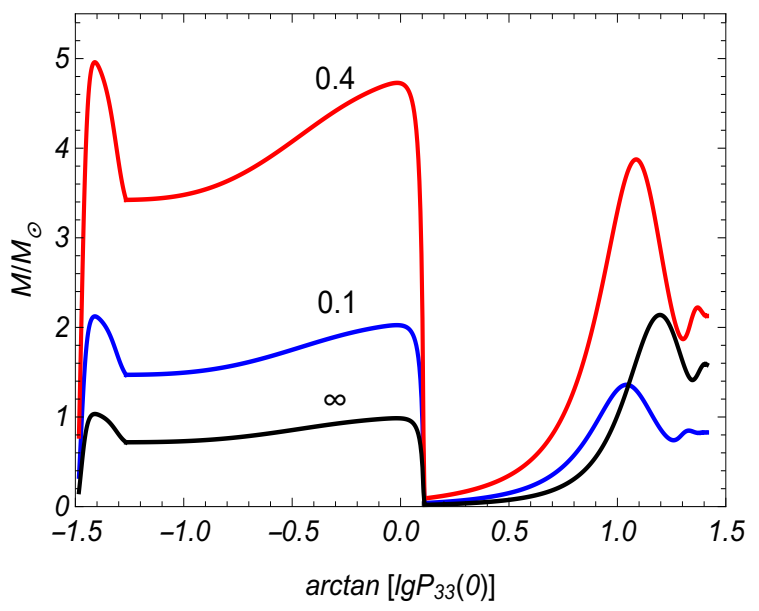

Figure 1: The mass of a superdense star as a function of the central pressure $P(0)$. The numbers near the curves correspond to the values of the BSTT parameter $\zeta$ and $P_{33}=P / 10^{33} \mathrm{erg} \cdot \mathrm{cm}^{-3}$. The curve $\zeta=\infty$ corresponds to configurations in general relativity.

In general relativity, the configurations corresponding to the monotonically increasing segment on the left of the first local maximum correspond to white dwarfs. The monotonically increasing segment between the first and second local maxima corresponds to configurations which are unstable against radial perturbations. The monotonically increasing segment between the second and third local maxima corresponds to neutron stars. Note that in BSTT the issue of stability of the static configurations requires an additional investigation. As seen from the graphs, depending on the value of the theory parameter $\zeta$, in BSTT the masses of the corresponding configurations for a fixed value of the pressure at the center can be essentially larger compared with the corresponding values in general relativity. For large values of the parameter, $\zeta \gg 1$, the curves in BSTT and in general relativity are practically indistinguishable. Note that the study of neutron star masses, presently has attracted a considerable interest both in the fields of theory and observations (see, for instance, Ozel \& Freire (2016), Horvath et al. (2017)). This, in particular, was motivated by the observation of neutron stars with masses near $2 M_{\odot}$. 
In addition to the mass of the configuration, the external geometry is determined by $\beta \equiv \beta\left(r_{1}\right)$. In terms of this parameter one has $\alpha=$ $3 \beta / M$. In figure 2 we have plotted the ratio $\beta / M_{\odot}$ as a function of $P(0)$ for $\zeta=0.1,0.4, \infty$. For configurations corresponding to white dwarfs one has $\beta / M_{\odot} \ll 1$. The numerical analysis shows that the difference of the external solution from that in general relativity with the same value of the mass is rather small. The numerical integration shows that for $\zeta>2$ the parameters of the configurations in BSTT are very close to those for general relativity.

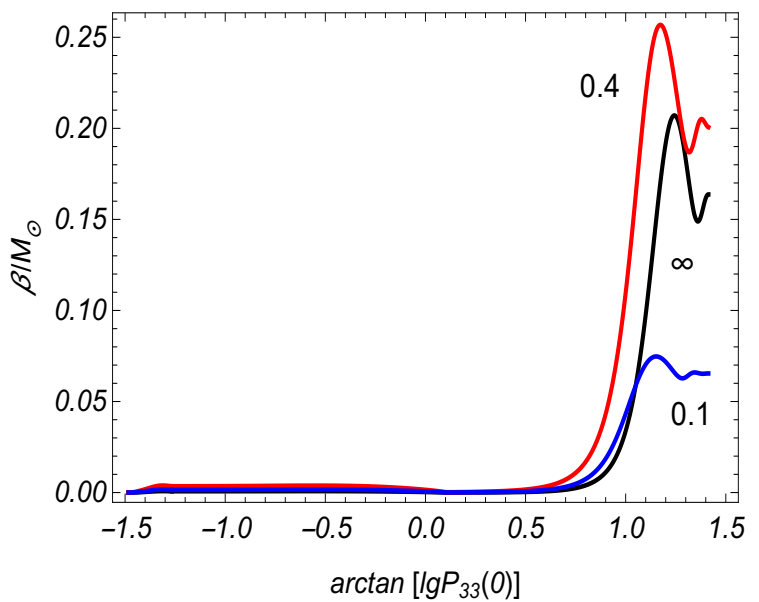

Figure 2: The dependence of the ratio $\beta / M_{\odot}$ on the central pressure. The numbers near the curves correspond to the values of the BSTT parameter $\zeta$.

Figure 3 presents the radius of the configuration versus the central pressure for different values of the parameter $\zeta$ (numbers near the curves). The graphs show that, depending on the value of $\zeta$, the radii of the configurations may notably exceed the corresponding values in general relativity.

\section{Conclusion}

We have investigated static spherically symmetric configurations of gravitating masses in the simplest version of BSTT with a constant coupling function and with the zero cosmological function. The constraints on the coupling constant, obtained from the observations within the framework of the post-Newtonian approximation, are essentially weaker than those in usual scalar-tensor theories (Brans-Dicke theory). The corresponding external solution is given by (13), (14) and (16). In addition to the mass, the solution depends on the parameter $\alpha$ that is determined in terms of the integral involving the pressure of the gravitating matter. For the integration 


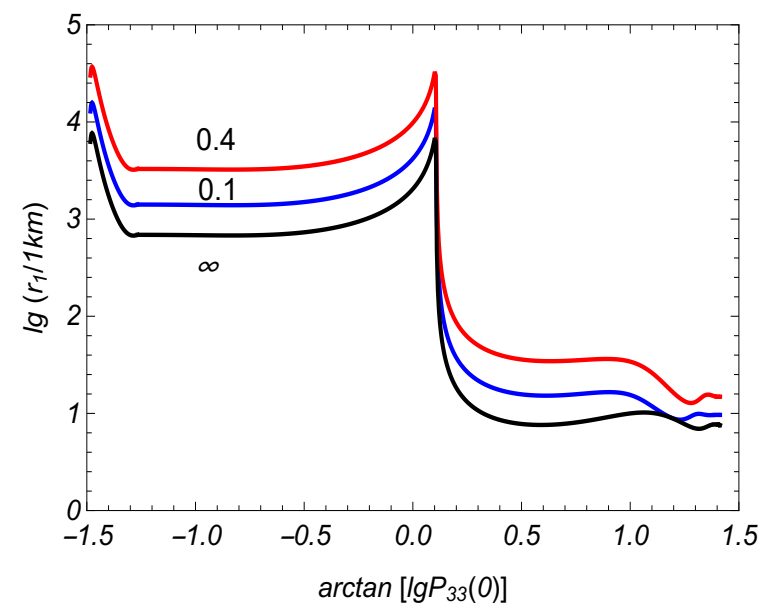

Figure 3: The radius of a superdense star versus the central pressure. The numbers near the curves correspond to the values of the parameter $\zeta$. The curve $\zeta=\infty$ corresponds to the results in general relativity.

of the field equations inside the configuration we have used the equation of state from Sahakian (1974, 1995) and Grigorian \& Sahakian (1983). The corresponding numerical data are presented in figures 1-3. These results show that, for strong gravitational fields, depending on the value of the BSTT parameter $\zeta$ the characteristics of the superdense configurations may essentially differ from those in general relativity. Note that we have considered spherically symmetric solutions with variable gravitational scalar.

\section{References}

Avakian, M.R.; Grigorian, L.Sh.; Saharian, A.A. 1990, Astrophysics, 35,121

Avakian, M.R.; Grigorian, L.Sh.; Saharian, A.A. 1991, Astrophysics, 34, 265

Babak, S.V.; Grishchuk, L.P. 1999, Phys. Rev. D, 61, 024038

Clifton, T.; Ferreira, P.G.; Padilla, A.; Skordis, C. 2012, Modified gravity and cosmology. Phys. Rep., 513, 1-189

Fujii, Y.; Maeda, K. 2009 The Scalar-Tensor Theory of Gravitation, Cambridge University Press, England

Grigorian, L.Sh.; Khachatryan, H.F.; Saharian, A.A. 2018, Models of compact stars in the Bimetric scalar-tensor theory of gravitation. Particles, 1, 203

Grigorian, L.Sh.; Sahakian, G.S. 1983, Astrophys. Space Sci., 95, 305

Grigorian, L.Sh.; Saharian, A.A. 1990a, Astrophysics, 31, 359 
Grigorian, L.Sh.; Saharian, A.A. 1990b, Astrophys. Space Sci., 167, 271

Grigorian, L.Sh.; Saharian, A.A. 1990c, Astrophysics, 32, 491

Grigorian, L.Sh.; Saharian, A.A. 1991, Astrophys. Space Sci., 180, 39

Grigorian, L.Sh.; Saharian, A.A. 1990d, Astrophysics, 33, 107

Grigorian, L.Sh.; Saharian, A.A. 1994, Astrophysics, 37, 167

Hinterbichler, K. 2012, Theoretical aspects of massive gravity, Rev. Mod. Phys., 84, 671

Horvath, J.E.; Valentim, R. The Masses of Neutron Stars. In: Alsabti A.; Murdin P. (eds) Handbook of Supernovae; Springer: Cham, 2017.

Özel, F.; Freire, P. 2016, Annu. Rev. Astron. Astrophys., 54, 401

Sahakian, G.S. 1974, Equilibrium Configurations of Degenerate Gaseous Masses; Halsted (Wiley): New York, Israel Program for Scientific Translations, Jerusalem, 1974.

Sahakian, G.S. Physics of Neutron Stars; JINR Publishing Department: Dubna, Russia, 1995.

Saharian, A.A. 1993a, Astrophysics, 36, 423

Saharian, A.A. 1993b, Astrophysics, 36, 603

Schmidt-May, A.; von Strauss, M. 2016, Recent developments in bimetric theory. J. Phys. A: Math. Theor., 49, 183001

Will, C.M. 2018 Theory and Experiment in Gravitational Physics, Cambridge University Press, England 